\title{
ESTRUTURA DA COMUNIDADE DE PEIXES DEMERSAIS DA BAÍA DE SEPETIBA, RJ
}

\author{
FRANCISCO GERSON ARAÚJO, ${ }^{1}$ ANTÔNIO GOMES DA CRUZ-FILHO, ${ }^{2}$ \\ MÁRCIA CRISTINA COSTA DE AZEVÊDO ${ }^{1} \mathrm{e}$ \\ ALEXANDRE CLÍSTENES DE ALCÂNTARA SANTOS ${ }^{3}$ \\ ${ }^{1}$ Universidade Federal Rural do Rio de Janeiro, Posto de Aqüicultura, Laboratório de \\ Ecologia de Peixes, Km 47 da Antiga Rodovia Rio-São Paulo, CEP 23851-970, Seropédica, RJ \\ ${ }^{2}$ Fundação Instituto de Pesca do Estado do Rio de Janeiro, Praça XV de Novembro, 4, \\ CEP 20010-010, Rio de Janeiro, RJ \\ ${ }^{3}$ Universidade Estadual de Feira de Santana, Departamento de Ciências Biológicas, \\ Campus Universitário, CEP 44031-460, Feira de Santana, BA \\ Correspondência para: Francisco Gerson Araújo, Rua Jorge Rudge, 89/506A, Vila Isabel, \\ CEP 20550-220, Rio de Janeiro, RJ \\ Recebido em 13/06/97 - Aceito em 27/05/98 - Distribuído em 28/08/98
}

(Com 8 figuras)

\begin{abstract}
Community structure of the demersal fish of Sepetiba Bay, RJ

A year-long otter trawl survey in the Sepetiba Bay, Rio de Janeiro State, Brazil, was carried out in order to describe the fish community structure and their spatial and seasonal variations. Sampling carried out between July 1993 and June 1994, yielded 97 species, 70 genera and 38 families. Ariidae, Gerreidae, Sciaenidae, Carangidae and Sparidae families amounted 69,9\% of the total catch in number, and Ariidae, Sparidae, Gerreidae, Haemulidae and Sciaenidae amounted 67,9\% of the total weight. Genidens genidens, Gerres aprion, Cathorops spixii, Micropogonias furnieri and Chloroscombrus chrysurus were the most numerous species, each one contributing more than $5 \%$ of the total catches. Higher numbers of fishes were associated with low depths and transparencies of the inner Bay, while salinity is a quite stable environmental parameter ranging around $29 \%$ o most of the time all over the Bay. Numbers of fish are higher in Late Summer and Early Winter. Overall, most number and weight of fishes were higher in the inner Bay than in the outer area next to the sea limit, while the number of species was higher in the outer Bay. The high number of fish species suggests the important role played by the Sepetiba Bay as rearing and residence grounds for the fish community.
\end{abstract}

Key words: Sepetiba Bay, ichthyofauna, coastal lagoons, fish, community.

\section{RESUMO}

Um programa de amostragens mensais de arrasto de fundo em sete estações de coleta na Baía de Sepetiba foi realizado entre julho de 1993 e junho de 1994 com o objetivo de descrever a estrutura da comunidade de peixes e suas variações espaciais e temporais. Noventa e sete espécies de peixes foram levantadas, compreendendo 70 gêneros e 38 famílias. As famílias Ariidae, Gerreidae, Sciaenidae, Carangidae e Sparidae, nesta ordem, foram as mais abundantes em número, contribuindo com 69,9\% do total capturado, enquanto Ariidae, Sparidae, Gerreidae, Haemulidae e Sciaenidae somaram 67,9\% do peso total. Genidens genidens, Gerres aprion, Cathorops spixii, Micropogonias furnieri e Chloroscombrus chrysurus, nesta ordem, foram as espécies mais abundantes, contribuindo cada uma com mais de $5 \%$ do número total de peixes capturados. O maior número de peixes foi associado às menores profundidades e transparências, enquanto a salinidade apresentou, na maior parte do tempo, valores estáveis em torno de $29 \%$ o. Maiores abundâncias de peixes ocorreram entre fins do verão e início do inverno. O número e peso dos peixes foram maiores na zona interna da Baía enquanto 
o número de espécies foi maior na zona externa, próxima ao limite com o mar. O elevado número de espécies de peixes sugere o importante papel da Baía de Sepetiba como área de criação e desenvolvimento para a comunidade de peixes.

Palavras-chave: Baía de Sepetiba, ictiofauna, lagoas costeiras, peixes, comunidade.

\section{INTRODUÇÃO}

Lagunas costeiras e baías desempenham importante papel como áreas de desova, criação e refúgio para muitas espécies de peixes, e este fato tem sido amplamente documentado na literatura através da descrição da composição e estrutura, bem como dos padrões de variações espaciais e temporais, dos grupos mais abundantes (Livingston et al., 1976; Amezcua-Linares \& Yañez-Arancibia, 1980; Cunningham, 1983; Hook, 1991; Wright, 1991; Monteiro-Neto et al., 1990; Blaber et al., 1992; Sedberry \& Carter, 1993; Villarroel, 1994). Na costa do Rio de Janeiro, dentre alguns trabalhos realizados sobre a comunidade de peixes de zonas lagunares, citam-se os exemplos das Lagoas Tijuca-Jacarepaguá-Marapendi (Volcker \& Andreata, 1982; Andreata et al., 1989, 1990), Sistema Lagunar Itaipu-Piratininga (Silva, 1994) e Sistema Lagunar de Maricá (Brum et al., 1994).

A Baía de Sepetiba é considerada um dos mais importantes ecossistemas aquáticos do estado do Rio de Janeiro, por constituir área de criação de peixes e crustáceos de importância econômica local (Costa, 1992).

Com o recente desenvolvimento do complexo industrial e a crescente densidade populacional nas zonas urbanas situadas nas proximidades da Baía, inúmeras alterações na qualidade de água e sua transferência para a biota têm sido descritas por Pfeiffer et al. (1985).

Um levantamento preliminar da comunidade de peixes e crustáceos decápodes foi realizado por Oshiro \& Araújo (1987) e estudos da biologia de alguns grupos de peixes foram feitos por Oliveira (1988) e por Souza \& Araújo (1990). Mais recentemente, um estudo de sinecologia da ictiofauna foi realizado por Cruz-Filho (1995) e por Araújo et al. (1997).

O objetivo do presente trabalho é descrever a composição e estrutura da comunidade de peixes demersais da Baía, analisar os padrões temporais e espaciais e as variações na ocorrência dos grupos mais abundantes.

\section{MATERIAL E MÉTODOS}

\section{Área de estudo}

A Baía de Sepetiba (Latitude: 2254' a $23^{\circ} 04^{\prime}$ 'S ; Longitude: $43^{\circ} 34^{\prime}$ a $44^{\circ} 10^{\prime} \mathrm{W}$ ) localizase no Estado do Rio de Janeiro e apresenta área de $305 \mathrm{Km}^{2}$. Sua forma é alongada, limitando-se a Norte e a Leste pelo continente, ao Sul pela Restinga de Marambaia e a Oeste pela Baía de Ilha Grande (Fig. 1). Seu maior comprimento é de 43 $\mathrm{Km}$ no sentido Este-Oeste e sua maior largura é de $17 \mathrm{Km}$ no sentido Norte-Sul, com profundidade máxima de $30 \mathrm{~m}$ próxima à Ilha de Itacuruçá, porém $40 \%$ de sua área apresenta profundidade média de 5 m (Coelho e Carvalho, 1973).

Vários rios de pequenos volumes de água e canais de drenagem desaguam na Baía, diluindo águas e trazendo aluviões e efluentes de poluentes diversos, oriundos de áreas domésticas e industriais dos municípios do Rio de Janeiro, Itaguaí e Mangaratiba.

A maior contribuição de água doce para a Baía é proveniente do Canal de São Francisco (Rio Guandu), que carreia águas originalmente desviadas do rio Paraíba do Sul pela LIGHT Serviços de Eletricidade S/A, que são utilizadas na geração de energia hidroelétrica nas usinas daquela empresa, localizadas no município de Piraí, RJ. O fundo da Baía é principalmente lodoso, na maior parte da área interna, com formações de silte, argila e poucas áreas de areia e cascalho na parte mais externa e próxima da ligação com o mar (Costa, 1992).

\section{Programa de amostragem}

Um programa de amostragem mensal com arrastos de fundo, foi realizado entre julho de 1993 e junho de 1994, compreendendo sete estações de coletas distribuídas no interior da Baía, tanto na parte mais interna e protegida pelo "cordão" de ilhas, como na mais externa e desprotegida próxima da ligação com o mar. Foram amostradas as seguintes estações de coletas, numeradas na ordem de distribução espacial da área mais exter- 
na para a área mais interna da Baía, e identificadas conforme o nome da localidade (Fig. 1). A escolha das estações foi feita com base em sua localização geográfica, visando cobrir toda a área da Baía, com locais de amostragens tanto na área mais externa (estações 1 a 3 ) como na mais interna (estações 4 a 7).

As coletas foram realizadas com barco "arrasteiro" de $12 \mathrm{~m}$ de comprimento, do tipo "balão", utilizado comumente na pesca praticada no interior da Baía, provido com rede de arrasto com portas, tralha superior de $10,5 \mathrm{~m}$, tralha inferior de $12 \mathrm{~m}$, malha de $25 \mathrm{~mm}$ de distância entre nós consecutivos nas asas e $15 \mathrm{~mm}$ no ensacador. As amostragens foram realizadas num período de aproximadamente 14 horas, tendo sido iniciadas aproximadamente às 6 horas, e a seqüência de amostragens nas estações foi aleatória. Cada arrasto teve duração padronizada de meia hora e velocidade de aproximadamente 2 nós, cobrindo uma extensão de aproximadamente $1 \mathrm{Km}$. Em cada amostragem, foram registradas: a transparência da água, utilizando um Disco de Secchi com graduação em $\mathrm{cm}$; profundidade, utilizando uma cabo graduado em $\mathrm{cm}$ provido de poita de $7 \mathrm{Kg}$; temperatura da água de superfície e de fundo, usando um termômetro de imersão com coluna de mercúrio, com precisão de $0,1^{\circ} \mathrm{C}$; e salinidade de superfície e de fundo, usando um refratômetro ótico marca Erma Tokio, com precisão de 0,5 parte por mil (\%o). Todos os peixes foram identificados, contados e pesados por espécie. Parte do material coletado foi fixado em formol a $10 \%$, e conservado em álcool $70 \%$. Os peixes foram identificados, com o auxílio dos trabalhos de Norman (1934), Figueiredo (1977), Figueiredo \& Menezes (1978, 1980), Fischer (1978), Menezes \& Figueiredo $(1980,1985)$ e Andreata $(1987,1988)$.

\section{ANÁLISE DOS DADOS}

Para avaliação das variações espaciais e temporais foi aplicada Análise Bifatorial de Variância sobre os dados de abundância dos peixes, expressa em CPUE (Captura por Unidade de Esforço = número de indivíduos por amostra), e dos fatores ambientais. A normalidade e a homocedasticidade das variáveis, tanto dos dados bióticos como dos fatores ambientais, foram determinadas através do teste de Bartlet (Sokal \& Rohlf, 1981), e como foi encontrado que a maioria dos dados não atendia a esses requisitos, utilizou-se a transformação logarítmica $\log (x+1)$ para os dados bióticos e $\log (\mathrm{x})$ para os dados abióticos, em que Log é o logaritmo na base $10 \mathrm{e} x$ é o valor não transformado. Tais transformações antecederam a Análise de Variância, a qual foi seguida do teste de diferenças de médias de Tukey em nível de confiança de $95 \%(\mathrm{p}<0,05)$, para determinação de quais médias eram significativamente diferentes, sempre que a hipótese zero era rejeitada.

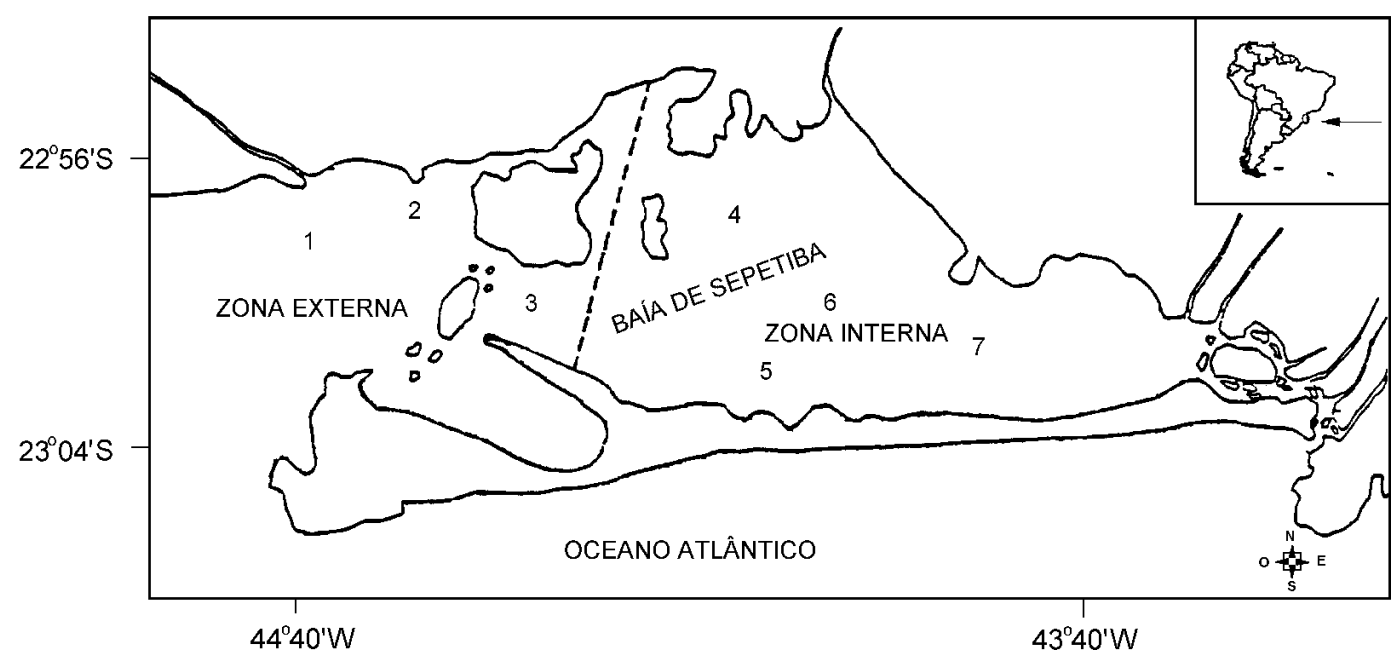

Fig. 1 - Baía de Sepetiba, RJ, com a indicação das estações de coleta. Zona interna: 1 - Laje das Enxadas, 2 - Ilha do Socó, 3 - Ilha Bonita. Zona externa: 4 - Costa do Guandu, 5 - Canal da Restinga, 6 - Meio da Baía e 7 - Fundo da Baía. 
As variações temporais foram analisadas mensalmente, incluindo as diferentes estações do ano: verão (dezembro, janeiro e fevereiro), outono (março, abril e maio), inverno (junho, julho e agosto) e primavera (setembro, outubro e novembro). Similaridades entre as estações em termos de composição da ictiofauna foram medidas com o coeficiente de Czekanowski (Bray \& Curtis, 1957).

\section{RESULTADOS}

\section{Parâmetros ambientais}

Diferenças sazonais significativas foram verificadas para a temperatura da água de superfície e de fundo e para a transparência (ANOVA, $p<0,05)$, enquanto variações espaciais foram apresentadas para a salinidade da água de superfície, transparência e profundidade, com os valores de $\mathrm{F}$ tendo sido altamente significativos (Tabela 1).

A temperatura da água de superfície e de fundo apresentou um evidente padrão de variação sazonal com mínimas de aproximadamente $22^{\circ} \mathrm{C}$ em agosto e máximas em torno de $26^{\circ} \mathrm{C}$ em fevereiro (Fig. 2). Não foram apresentadas diferenças significativas $(p>0,01)$ na temperatura da água entre as estações de coletas (Fig. 3). A temperatura da água de fundo esteve sempre entre 1 e $2^{\circ} \mathrm{C}$ abaixo da temperatura da água de superfície, com exceção de maio e junho, quando esta situação se inverteu (Fig. 2).

A salinidade da água de fundo apresentou pequena amplitude de variação, oscilando entre
$29 \%$ e $32 \%$ o, sem evidenciar uma tendência ao longo do ciclo anual, enquanto a salinidade na superfície apresentou sempre valores abaixo da salinidade no fundo com diferenças nas médias mensais de até 5\%o (Figs. 2 e 3).

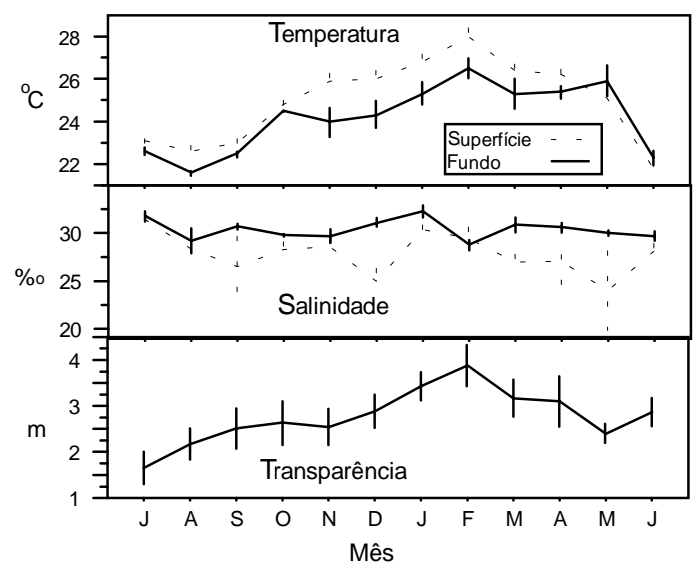

Fig. 2 - Variação temporal da temperatura, salinidade e transparência da água na Baía de Sepetiba, RJ, entre julho de 1993 e junho de 1994. Barras representando +/- 1 erro padrão.

Espacialmente, apenas a salinidade da água de superfície apresentou menores médias na estação 4, Costa do Guandu (média $=21,1 \%$ ), em relação às outras estações (médias: 28,3-29,9\%o). As únicas interações (estações do ano x locais de coleta) significativas foram apresentadas para a sa-linidade na superfície, o que indica que diferenças sazonais apesar de não significativas foram mais amplas apenas na estação de Costa do Guandu (estação 4), devido à maior influência de

TABELA 1

Valores significativos $(p<0,05)$ de $F$ para análise de variância bifatorial sobre os fatores ambientais na Baía de Sepetiba, RJ.

\begin{tabular}{|c|c|c|c|}
\hline \multirow[t]{2}{*}{ Fatores } & \multicolumn{2}{|c|}{ Efeitos principais } & \multirow{2}{*}{$\begin{array}{c}\text { Interação } \\
\begin{array}{c}\text { Est. do ano x locais } \\
\text { g. } 1 .=18\end{array}\end{array}$} \\
\hline & $\begin{array}{c}\text { Estação do ano } \\
\text { g. } 1 .=3\end{array}$ & $\begin{array}{l}\text { Locais } \\
\text { g. } 1 .=6\end{array}$ & \\
\hline Temperatura superfície & $41,9 * *$ & n.s. & n.s. \\
\hline Temperatura fundo & $24,2 * *$ & n.s. & n.s. \\
\hline Salinidade superfície & n.s. & $7,2 * *$ & $2,1^{*}$ \\
\hline Salinidade fundo & n.s. & n.s. & n.s. \\
\hline Transparência & $6,3^{* *}$ & $15,0 * *$ & n.s. \\
\hline Profundidade & - & 31,2 & - \\
\hline
\end{tabular}

Níveis de significância: $* \mathrm{p}<0,05 ; * * \mathrm{p}<0,01$.

g.l. - graus de liberdade; n.s. - não significativo. 
água doce, especialmente no mês de maio, o que aumentou a variabilidade da média.

A transparência e a profundidade apresentaram o mesmo padrão espacial, com maiores valores nas estações da zona externa da Baía e próximas ao limite com o mar (estações 1 a 3 ), e menores valores nas estações mais internas da Baía (estações 4 a 7), principalmente Costa do Guandu e Fundo da Baía. Sazonalmente, a transparência foi maior no verão e outono, principalmente entre dezembro e abril, e menores entre maio e novembro (Figs. 2 e 3 ).

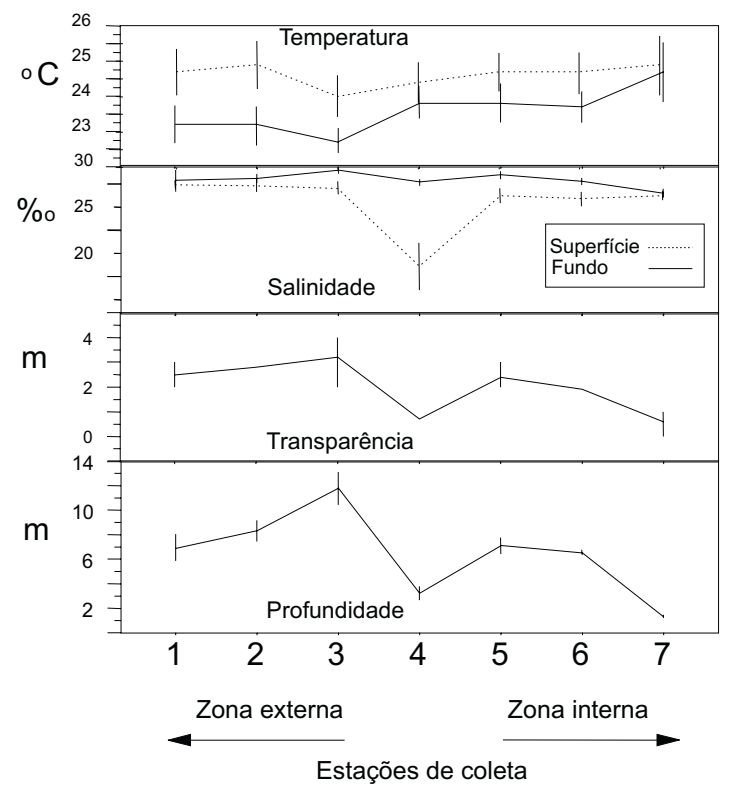

Fig. 3 - Variação espacial da temperatura, salinidade e transparência da água na Baía de Sepetiba, RJ, entre julho de 1993 e junho de 1994. Barras representando +/- 1 erro padrão.

\section{Composição e estrutura}

Nas 75 amostragens, foram capturadas 97 espécies de peixes, compreendendo 70 gêneros e 38 famílias e totalizando 22.342 indivíduos e 840,76 $\mathrm{Kg}$ de peixes (Tabela 2). As famílias Ariidae, Gerreidae, Sciaenidae, Carangidae e Sparidae, nesta ordem, apresentaram as maiores abundâncias numéricas, contribuindo cada uma com mais de 5\% do total de peixes e somando $69,9 \%$ do total capturado. Em relação ao peso, Ariidae, Sparidae, Gerreidae, Haemulidae e Sciaenidae contribuíram, cada uma, com mais de 5\% do total de peixes e somaram $67,9 \%$ do total capturado (Fig. 4).

Cinco espécies, sendo duas Ariidae (G. genidens e C. spixii), uma Gerreidae (G. aprion), uma Sciaenidae (M. furnieri) e uma Carangidae (C. chrysurus), representaram $46,8 \%$ do número total de peixes, enquanto três Ariidae ( $G$. genidens, C. spixii e Sciadeichthys luniscutis), uma Sparidae (Archosargus rhomboidalis) e uma Haemulidae (Haemulon steindachneri) representaram $42,7 \%$ do peso total capturado (Fig. 5), tendo cada uma destas espécies contribuído com mais de 5\% do total de peixes capturados.

Das 97 espécies registradas, 22 representaram $91,5 \%$ da captura total em número, sendo que as 75 espécies restantes representaram menos de 9\% do total das capturas, cada uma delas contribuindo, individualmente, com menos de $1 \%$ do total de peixes. Quinze espécies foram registradas uma única vez nas capturas, contribuindo com um único indivíduo (Tabela 2).

Em relação às freqüências de ocorrência, das cinco espécies mais numerosas ( $G$. genidens, G. aprion, C. spixii, M. furnieri e C. chrysurus), apenas $G$. aprion apresentou maior constância nas capturas, com mais de $75 \%$ de presença nas amostras, juntamente com Prionotus punctatus, Diplectrum radiale e Etropus longimanus, espécies de grande constância, porém de menor abundância numérica. Vinte e duas espécies apareceram apenas uma vez nas capturas (Tabela 2).
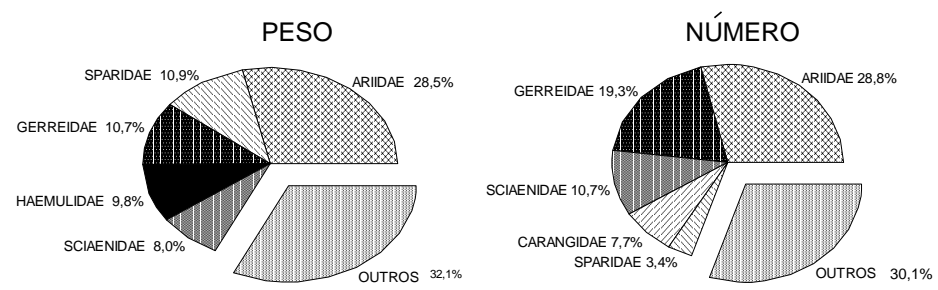

Fig. 4 - Percentual, em número e peso, das famílias de peixes mais abundantes na Baía de Sepetiba, RJ, entre julho de 1993 e junho de 1994. 
TABELA 2

Número total de peixes capturados na Baía de Sepetiba, RJ, entre julho 1993 e junho 1994, sumarizados por famílias. FO = freqüências de ocorrência.

\begin{tabular}{|c|c|c|c|c|c|}
\hline Famílias & Espécies & $\begin{array}{l}\text { № de ordem } \\
\text { le abundância }\end{array}$ & $\begin{array}{c}\text { № de peixes } \\
\text { coletados }\end{array}$ & $\begin{array}{l}\% \text { do } \\
\text { total }\end{array}$ & $\begin{array}{c}\text { FO } \\
\%\end{array}$ \\
\hline TRIAKIDAE & Galeorhinus vitaminicus (Buen, 1950) & 83 & 1 & $<1$ & 1,3 \\
\hline RHINOPTERIDAE & Rhinoptera bonasus (Mitchill, 1815) & 79 & 4 & $<1$ & 4,0 \\
\hline RHINOBATIDAE & Rhinobatus percellens (Walbaum, 1792) & 59 & 8 & $<1$ & 6,6 \\
\hline \multirow[t]{2}{*}{ DASYATIDAE } & Dasyatis centroura (Mitchill, 1815) & 74 & 2 & $<1$ & 1,3 \\
\hline & Dasyatis guttata (Bloch \& Schneider, 1808) & 58 & 9 & $<1$ & 9,3 \\
\hline GYMNURIDAE & Gymnura altavela (Linnaeus, 1758) & 71 & 3 & $<1$ & 2,6 \\
\hline MURAENIDAE & Gymnothorax ocellatus (Agassix, 1831) & 37 & 45 & $<1$ & 16,0 \\
\hline \multirow[t]{4}{*}{ CLUPEIDAE } & Harengula clupeola (Cuvier, 1829) & 28 & 69 & $<1$ & 17,3 \\
\hline & Opisthonema oglinum (Lesueur, 1818) & 62 & 6 & $<1$ & 5,3 \\
\hline & Pellona harroweri (Fowler, 1917) & 27 & 99 & $<1$ & 4,0 \\
\hline & Sardinella brasiliensis (Steindachner, 1780) & 41 & 36 & $<1$ & 4,0 \\
\hline \multirow[t]{4}{*}{ ENGRAULIDIDAE } & Anchoa januaria (Steindachner, 1879) & 47 & 22 & $<1$ & 8,0 \\
\hline & Anchoa tricolor (Agassiz, 1829) & 15 & 557 & 2,49 & 41,3 \\
\hline & Anchoviella brevirostris (Gunther, 1869) & 83 & 1 & $<1$ & 1,3 \\
\hline & Centengraulis edentulus (Cuvier, 1828) & 21 & 329 & 1,47 & 21,3 \\
\hline \multirow[t]{5}{*}{ ARIIDAE } & Bagre marinus (Mitchill, 1814) & 83 & 1 & $<1$ & 1,3 \\
\hline & Cathorops spixii (Agassiz, 1829) & 3 & 1418 & 6,35 & 34,7 \\
\hline & Genidens genidens (Mitchill, 1814) & 1 & 4204 & 18,82 & 64,0 \\
\hline & Netuma barba (Lacépede, 1803) & 20 & 334 & 1,49 & 21,3 \\
\hline & Sciadeichthys luniscutis (Valenciennes, 1837) & 18 & 468 & 2,09 & 38,6 \\
\hline SYNODONTIDAE & Synodus foetens (Linnaeus, 1766) & 39 & 40 & $<1$ & 21,3 \\
\hline BATRACHOIDIDAE & Porichthys porosissimus (Valenciennes, 1837) & 53 & 12 & $<1$ & 8,0 \\
\hline \multirow[t]{2}{*}{ SCORPAENIDAE } & Scorpaena isthmensis (Meek \& Hildebrand, 1928) & 65 & 4 & $<1$ & 5,3 \\
\hline & Scorpaena plumieri $($ Bloch, 1797) & 95 & 1 & $<1$ & 1,3 \\
\hline TRIGLIDAE & Prionotus punctatus (Bloch, 1797) & 14 & 557 & 2,49 & 88,0 \\
\hline DACTYLOPTERIDAE & Dactylopterus volitans (Linnaeus, 1758) & 60 & 7 & $<1$ & 8,0 \\
\hline CENTROPOMIDAE & Centropomus parallelus (Poey, 1860) & 83 & 1 & $<1$ & 1,3 \\
\hline \multirow[t]{3}{*}{ SERRANIDAE } & Diplectrum formosum (Linnaeus, 1766) & 51 & 13 & $<1$ & 4,0 \\
\hline & Diplectrum radiale (Quoy \& Gaimard, 1824) & 16 & 526 & 2,35 & 76,0 \\
\hline & Epinephelus niveatus (Valenciennes, 1828) & 66 & 3 & $<1$ & 2,6 \\
\hline PRIACANTHIDAE & Priacantus arenatus (Cuvier, 1829) & 83 & 1 & $<1$ & 1,3 \\
\hline POMATOMIDAE & Pomatomus saltator (Linnaeus, 1766) & 35 & 47 & $<1$ & 14,6 \\
\hline \multirow[t]{10}{*}{ CARANGIDAE } & Caranx crysos (Mitchill, 1815) & 83 & 1 & $<1$ & 1,3 \\
\hline & Caranx hippos (Linnaeus, 1766) & 66 & 3 & $<1$ & 4,0 \\
\hline & Chloroscombrus chrysurus (Linnaeus, 1766) & 5 & 1276 & 5,71 & 64,0 \\
\hline & Oligoplites palometa (Cuvier, 1833) & 76 & 2 & $<1$ & 2,6 \\
\hline & Oligoplites saurus (Bloch \& Schneider, 1801) & 83 & 1 & $<1$ & 1,3 \\
\hline & Selene setapinnis (Mitchill, 1815) & 19 & 364 & 1,63 & 26,6 \\
\hline & Selene vomer (Linnaeus, 1758) & 55 & 10 & $<1$ & 6,6 \\
\hline & Selene cf. vomer (Linnaeus, 1758) & 29 & 69 & $<1$ & 10,3 \\
\hline & Selene sp. (Linnaeus, 1758) & 29 & 69 & $<1$ & 10,6 \\
\hline & Trachinotus falcatus (Linnaeus, 1758) & 92 & 1 & $<1$ & 1,3 \\
\hline LUTJANIDAE & Lutjanus synagris (Linnaeus, 1758) & 83 & 1 & $<1$ & 1,3 \\
\hline \multirow[t]{4}{*}{ GERREIDAE } & Diapterus rhombeus (Cuvier, 1929) & 6 & 1034 & 4,63 & 50,7 \\
\hline & Diapterus richii (Goode \& Bean, 1882) & 72 & 2 & $<1$ & 1,3 \\
\hline & Gerres aprion (Baird \& Gaimard, 1854) & 2 & 2261 & 10,12 & 78,4 \\
\hline & Gerres gula (Cuvier, 1839) & 7 & 928 & 4,15 & 52,0 \\
\hline
\end{tabular}


TABELA 2 (continuação)

\begin{tabular}{|c|c|c|c|c|c|}
\hline Famílias & Espécies & $\begin{array}{l}\text { № de ordem } \\
\text { e abundância }\end{array}$ & $\begin{array}{c}\text { № de peixes } \\
\text { coletados }\end{array}$ & $\begin{array}{l}\% \text { do } \\
\text { total }\end{array}$ & $\begin{array}{l}\text { FO } \\
\%\end{array}$ \\
\hline \multirow[t]{2}{*}{ GERREIDAE (cont.) } & Gerres lefroyi (Goode, 1874) & 45 & 25 & $<1$ & 2,6 \\
\hline & Gerres melanopterus (Bleeker, 1863) & 33 & 57 & $<1$ & 4,0 \\
\hline \multirow[t]{7}{*}{ HAEMULIDAE } & Anisotremus surinamensis (Bloch, 1791) & 83 & 1 & $<1$ & 1,3 \\
\hline & Boridia grossidens (Cuvier, 1830) & 72 & 2 & $<1$ & 1,3 \\
\hline & Conodon nobilis (Linnaeus, 1758) & 72 & 2 & $<1$ & 1,3 \\
\hline & Haemulum steidachneri (Jordan \& Gilbert, 1882) & 13 & 560 & 2,51 & 18,7 \\
\hline & Orthopristis ruber (Cuvier, 1830) & 17 & 515 & 2,31 & 73,3 \\
\hline & Pomadasys corvinaeformis (Steindachner, 1868) & 72 & 2 & $<1$ & 2,6 \\
\hline & Pomadasys ramosus (Poey, 1860) & 72 & 2 & $<1$ & 2,6 \\
\hline SPARIDAE & Archosargus rhomboidalis (Linnaeus, 1758) & 9 & 768 & 63,09 & 42,7 \\
\hline \multirow[t]{10}{*}{ SCIAENIDAE } & Ctenosciaena gracilicirrhus (Metzlaar, 1919) & 23 & 185 & $<1$ & 29,3 \\
\hline & Cynoscion acoupa (Lacépede, 1802) & 44 & 29 & $<1$ & 2,6 \\
\hline & Cynoscion jamaicensis (Vaillant \& Bocourt, 1883) & 63 & 6 & $<1$ & 1,3 \\
\hline & Cynoscion leiarchus (Cuvier, 1830) & 11 & 587 & 2,63 & 41,3 \\
\hline & Cynoscion microlepidotus (Cuvier, 1830) & 83 & 1 & $<1$ & 1,3 \\
\hline & Cynoscion striatus (Cuvier, 1829) & 57 & 9 & $<1$ & 1,3 \\
\hline & Isopisthus parvipinnis (Cuvier, 1830) & 30 & 67 & $<1$ & 4,0 \\
\hline & Menticirrhus americanus (Linnaeus, 1758) & 24 & 165 & $<1$ & 58,6 \\
\hline & Micropogonias furnieri (Desmarest, 1823) & 4 & 1291 & 41,07 & 68,0 \\
\hline & Paralonchurus brasiliensis (Steindachner, 1875) & 34 & 56 & $<1$ & 4,0 \\
\hline \multirow[t]{2}{*}{ MULIDAE } & Mulus argentinae (Hubbs \& Marini, 1935) & 78 & 2 & $<1$ & 1,3 \\
\hline & Upenaeus parvus (Poey, 1853) & 64 & 6 & $<1$ & 4,0 \\
\hline EPHIPPIDIDAE & Chaetodipterus faber (Broussonet, 1782) & 31 & 62 & $<1$ & 25,3 \\
\hline MUGILIDAE & Mugil curema (Valenciennes, 1836) & 83 & 1 & $<1$ & 1,3 \\
\hline \multirow[t]{4}{*}{ SPHYRAENIDAE } & Sphyraena guachancho (Cuvier, 1829) & 66 & 3 & $<1$ & 2,6 \\
\hline & Sphyraena sphyraena (Linnaeus, 1758) & 83 & 1 & $<1$ & 1,3 \\
\hline & Sphyraena tome (Fowler, 1903) & 61 & 6 & $<1$ & 6,6 \\
\hline & Sphyraena sp (Röse, 1793) & 42 & 31 & $<1$ & 8,0 \\
\hline GOBIIDAE & Gobionellus oceanicus (Pallas, 1770) & 42 & 34 & $<1$ & 13,3 \\
\hline TRICHIURIDAE & Trichiurus lepturus (Linnaeus, 1758) & 32 & 61 & $<1$ & 18,6 \\
\hline STROMATEIDAE & Peprilus paru (Block, 1787) & 40 & 38 & $<1$ & 9,3 \\
\hline \multirow[t]{8}{*}{ BOTHIDAE } & Bothus ocellatus (Agassiz, 1829) & 54 & 11 & $<1$ & 5,3 \\
\hline & Citharichthys cornutus (Gunther, 1880) & 50 & 16 & $<1$ & 12,0 \\
\hline & Citharichthys spilopterus (Gunther, 1862) & 48 & 20 & $<1$ & 4,0 \\
\hline & Etropus longimanus (Norman, 1933) & 8 & 913 & 4,1 & 86,7 \\
\hline & Paralichthys bicyclophorus (Ribeiro, 1915) & 83 & 1 & $<1$ & 1,3 \\
\hline & Paralichthys orbignyana (Valenciennes, 1847) & 72 & 2 & $<1$ & 2,6 \\
\hline & Paralichthys triocellatus (Ribeiro, 1903) & 52 & 13 & $<1$ & 13,3 \\
\hline & Syacium papilossum (Linnaeus, 1758) & 66 & 3 & $<1$ & 2,6 \\
\hline SOLEIDAE & Achirus microphthalmus (Chabanaud, 1928) & 12 & 569 & 2,6 & 54,7 \\
\hline \multirow[t]{2}{*}{ CYNOGLOSSIDAE } & Symphurus plagusia (Block \& Schneider, 1801) & 22 & 267 & 1,2 & 66,6 \\
\hline & Symphurus symphurus (Quoy \& Gaimard, 1824) & 45 & 16 & $<1$ & 2,6 \\
\hline BALISTIDAE & Balistes capriscus (Gmelin, 1788) & 72 & 2 & $<1$ & 1,3 \\
\hline \multirow[t]{4}{*}{ TETRAODONTIDAE } & Lagocephalus laevigatus (Berg, 1895) & 46 & 24 & $<1$ & 8,0 \\
\hline & Sphoeroides greeleyi (Gilbert, 1900) & 10 & 707 & 3,2 & 36,0 \\
\hline & Sphoeroides testudineus (Linnaeus, 1758) & 25 & 162 & $<1$ & 32,0 \\
\hline & Sphoeroides sp (Linnaeus, 1758) & 26 & 157 & $<1$ & 22,6 \\
\hline \multirow[t]{2}{*}{ MONACANTHIDAE } & Monacanthus ciliatus (Mitchill, 1818) & 36 & 46 & $<1$ & 9,3 \\
\hline & Stephanolepis hispidus (Linnaeus, 1766) & 38 & 44 & $<1$ & 5,3 \\
\hline DIODONTIDAE & Chilomycterus spinosus (Linnaeus, 1758) & 56 & 10 & $<1$ & 6,6 \\
\hline
\end{tabular}




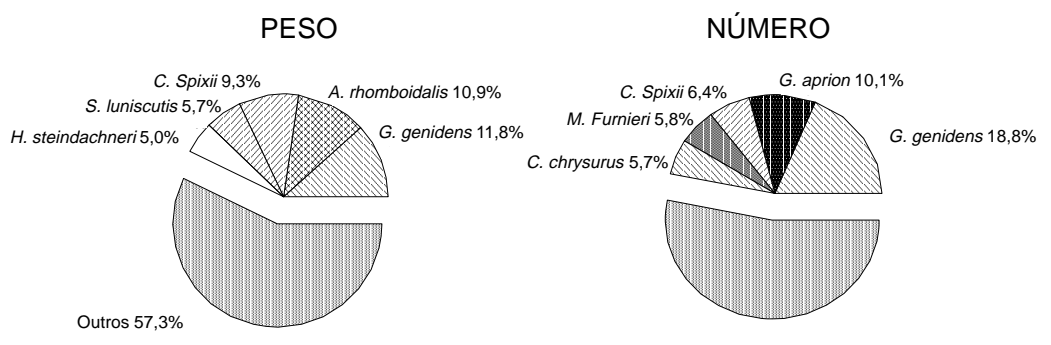

Fig. 5 - Percentual, em número e peso, das espécies de peixes mais abundantes na Baía de Sepetiba, RJ, entre julho de 1993 e junho de 1994.

\section{Variações espaciais e temporais}

Embora diferenças estatisticamente significativas em nível de $95 \%$ de confiança ( $p<0,05$ ) não tenham se verificado nas CPUEs, número de espécies/amostra e peso de peixes/amostra, a existência de um padrão diferenciado foi apresentado nestes indicadores da comunidade de peixes da Baía de Sepetiba (Tabela 3, Fig. 6). Maiores CPUEs e peso/amostra foram verificados nas estações de coletas mais internas da Baía e maiores número de espécie/amostra nas estações mais externas e próximas do limite com o mar.

A estação mais externa (estação 1) foi a que apresentou menor número total de indivíduos capturados (1.592) e a mais interna (estação 7) foi a que apresentou maior número (4.373). O número total de espécies foi maior (51-55) nas estações localizadas na zona externa (estações 1, 2 e 3) e menor (42-48) nas estações da zona interna (estações 5, 6 e 7). (Tabela 4.)

Dos grupos de peixes mais abundantes e que representarem mais de 5\% do número total capturado, G. aprion e M. furnieri apresentaram variações espaciais e sazonais significativas, enquanto G. genidens apresentou apenas variações espaciais ( $<<0,05$; Tabela 3$)$. De acordo com o teste de Tukey, G. genidens foi significativamente mais abundante nas estações 4 a 7 (zona interna), G. aprion, na estação 6 (meio da Baía) e $M$. furnieri, na estação 4 (Costa do Guandu). Também ainda de acordo com o mesmo teste estatístico, G. aprion foi significativamente mais abundante no outono, principalmente de março a junho, enquanto M. furnieri, no inverno e início da primavera, principalmente de julho a outubro (Tabela 3; Fig. 7). C. spixii foi mais abundante nos meses de outubro, fevereiro e abril, enquanto $C$. chrysurus, entre maio e agosto, sem apresentar diferenças sazonais nem espaciais em suas abundâncias.

Observaram-se indicações de separação espacial entre as espécies de maior abundância, com as grandes contribuições na abundância relativa (acima de 10\% do número total de peixes) tendo sido diferentes para as estações de coletas. $G$. genidens apresentou grande contribuição para as quatro estações da zona interna da Baía (estações 4, 5, 6 e 7), G. aprion apresentou-se amplamente distribuído, com grandes participações nas estações de coletas 2, 3, 5 e 6, C. spixii, nas estações 3 e 5, M. furnieri, na estação 1 e $C$. chrysurus, nas estações 4 e 5 (Tabela 4).

O coeficiente de similaridade de Czekanowski indicou maior semelhança da ictiofauna dentre as estações da zona interna (estações 1, 2 e 3 ) e as estações da zona externa (estações $3,4,5$ e 6 ) da Baía (Tabela 5), denotando uma mudança na ictiofauna da zona interna para a zona externa da Baía. As estações de Costa do Guandu (estação 4) e Ilha Bonita (estação 3) apresentaram a maior similaridade com as outras estações de coleta, tanto da zona interna como da zona externa da Baía, indicando ser uma área de transição na estrutura da comunidade de peixes.

As estações da zona interna foram numericamente dominadas por G. genidens e $C$. chrysurus, e as últimas, por M. furnieri e outras espécies de menor participação relativa, $\operatorname{com} G$. aprion e $C$. spixii sendo comuns em ambas as zonas da Baía. (Tabela 4.)

Médias mensais das CPUEs, embora não tenham apresentado diferenças significativas ( $p$ > $0,05)$, apresentaram valores comparativamente maiores de fevereiro a junho em relação aos demais meses do ano, enquanto o número de espécies por amostra não apresentou padrão sazonal 
definido, com maiores valores em outubro, fevereiro e julho, também não tendo apresentado diferenças significativas $(\mathrm{p}>0,05)$ entre os diferentes meses ou estações do ano. O número total de espécies registrado em cada mês em toda a Baía situou-se em torno de 40, com amplitude de variação de 30, em maio, até 45, em outubro e julho (Fig. 8).

O padrão anual de abundância (CPUE) apresentou pequena concordância entre as estações de coletas. Picos de abundâncias foram verificados para as estações Costa do Guandu (estação 4) em fevereiro, maio e junho, para Canal da Restinga (estação 5) em agosto, fevereiro e maio, para Meio da Baía (estação 6) em março e para Fundo da Baía (estação 7) em abril e junho. As estações da zona externa (estações 1, 2 e 3 ) não apresentaram sazonalidade na abundância da comunidade de peixes (Fig. 8).

O número total de espécies tem se mantido em torno de 20 para a maioria dos meses nas estações de coleta da zona externa da Baía (estações 1 a 3) e nas estações da zona interna 4 e 5; e em torno de 15 nas estações 6 e 7 da zona interna (Figura 8).

\section{TABELA 3}

Valores significativos $(\mathrm{p}<0,05)$ de $\mathrm{F}$ para análise de variância bifatorial sobre a abundância das cinco espécies de peixes mais numerosas na Baía de Sepetiba, RJ.

\begin{tabular}{lccc}
\hline Espécies & \multicolumn{2}{c}{ Efeitos principais } & Interação \\
\hline & $\begin{array}{c}\text { Estação do ano } \\
\text { g. } \mathbf{l} . \mathbf{=} \mathbf{3}\end{array}$ & $\begin{array}{c}\text { Locais } \\
\text { g. } \mathbf{l} . \mathbf{=} \mathbf{6}\end{array}$ & $\begin{array}{c}\text { Est. do ano } \mathbf{x} \text { locais } \\
\text { g. } \mathbf{l} . \mathbf{~} \mathbf{1 8}\end{array}$ \\
\hline G. genidens & n.s. & $7,5^{* *}$ & n.s. \\
G. aprion & $3,1^{*}$ & $5,8^{* *}$ & n.s. \\
C. spixii & n.s. & n.s. & n.s. \\
M. furnieri & $5.6^{* *}$ & $4.8^{* *}$ & $2,5^{*}$ \\
C. chrysurus & n.s. & n.s. & n.s. \\
CPUE & n.s. & n.s. & n.s. \\
SPP/amostra & n.s. & n.s. & n.s. \\
PESO/amostra & n.s. & n.s. & n.s. \\
\hline
\end{tabular}

Níveis de significância: *p $<0,05 ; * * p<0,01$. g. 1. - graus de liberdade n.s. - não significativo. SPP - número de espécies.

\section{TABELA 4}

Percentagens do total das capturas numéricas por estações de coleta para as espécies mais abundantes da Baía de Sepetiba, RJ. (Valores $>10 \%$ em negrito.)

\begin{tabular}{lccccccc}
\hline & \multicolumn{7}{c}{ Estações de coleta } \\
\hline Espécies & $\mathbf{1}$ & $\mathbf{2}$ & $\mathbf{3}$ & $\mathbf{4}$ & $\mathbf{5}$ & $\mathbf{6}$ & $\mathbf{7}$ \\
\hline G. genidens & 0,1 & 0,1 & 0,5 & $\mathbf{2 5 , 4}$ & $\mathbf{1 8 , 8}$ & $\mathbf{3 5 , 5}$ & $\mathbf{2 6 , 3}$ \\
G. aprion & 4,1 & $\mathbf{1 3 , 6}$ & $\mathbf{1 1 , 4}$ & 3,7 & $\mathbf{1 2 , 0}$ & $\mathbf{1 9 , 8}$ & 4,3 \\
C. spixii & 0,0 & 0,0 & $\mathbf{1 1 , 9}$ & 8,6 & $\mathbf{1 2 , 5}$ & 3,5 & 7,2 \\
M. furnieri & $\mathbf{1 6 , 1}$ & 2,4 & 0,5 & 7,4 & 1,8 & 5,4 & 8,8 \\
C. chrysurus & 0,4 & 2,4 & 1,7 & $\mathbf{1 2 , 2}$ & $\mathbf{1 3 , 2}$ & 0,9 & 5,1 \\
Outras espécies & 79,3 & 81,5 & 73,9 & 42,8 & 41,8 & 30,5 & 48,3 \\
\hline Total de peixes & 1.592 & 3.321 & 1.869 & 3.864 & 3.230 & 4.093 & 4.373 \\
Total de espécies & 51 & 52 & 55 & 50 & 48 & 42 & 45 \\
№ de amostras & 9 & 10 & 11 & 12 & 11 & 11 & 11 \\
\hline
\end{tabular}


TABELA 5

Coeficiente de Czekanowski expressando a similaridade da fauna e peixes entre as estações de coleta na Baía de Sepetiba, $R J$. (Valores $>0,35$ em negrito.)

\begin{tabular}{lcccccc}
\hline $\begin{array}{l}\text { Estações } \\
\text { de coleta }\end{array}$ & $\mathbf{1}$ & $\mathbf{2}$ & $\mathbf{3}$ & $\mathbf{4}$ & $\mathbf{5}$ & $\mathbf{6}$ \\
\hline 2 & $\mathbf{0 , 3 7}$ & & & & & \\
3 & $\mathbf{0 , 3 5}$ & $\mathbf{0 , 4 4}$ & & & & \\
4 & $\mathbf{0 , 3 6}$ & 0,28 & $\mathbf{0 , 3 9}$ & & & \\
5 & 0,31 & 0,33 & $\mathbf{0 , 4 9}$ & $\mathbf{0 , 6 8}$ & & \\
6 & 0,32 & 0,33 & $\mathbf{0 , 4 0}$ & $\mathbf{0 , 6 0}$ & $\mathbf{0 , 5 6}$ & $\mathbf{0 , 5 2}$ \\
7 & 0,24 & 0,20 & 0,25 & $\mathbf{0 , 6 1}$ & $\mathbf{0 , 4 8}$ & $\mathbf{0 , 5 2}$ \\
\hline
\end{tabular}

\section{DISCUSSÃO}

A ictiofauna demersal da Baía de Sepetiba é similar estruturalmente à ictiofauna de outras lagunas costeiras de zonas tropicais e subtropicais do Atlântico Oeste. Verificando a lista de espécies da Laguna dos Términos, México (YáñezArancibia et al., 1980), dos sistemas lagunares de Maricá (Brum et al., 1994) e Jacarepaguá (Andreata et al., 1990), está claro que Ariidae e Gerreidae são grupos de peixes amplamente distribuídos neste tipo de ambiente. Já em relação à Lagoa Joyuda, Porto Rico (Stoner, 1986), observou-se marcada diferenciação, especialmente pela dominância de Ariidae na Baía de Sepetiba e total ausência deste grupo de peixe naquela lagoa, com os fatores ambientais de temperatura e salinidade sendo muito semelhantes entre estes dois ambientes.

A família Ariidae, que contribuiu com a maior abundância em termos de número e peso de peixes na Baía de Sepetiba, foi substituída na Lagoa Joyuda pela família Soleidae, em que Achirus lineatus contribuiu com 32,9\% do número total de peixes capturados em um ciclo sazonal de coletas de arrasto de fundo entre agosto de 1984 e agosto de 1985; outro grupo de peixes dominante naquela lagoa foram os Gerreidae, também dominantes na Baía de Sepetiba.

Na Baía de Sepetiba, além de Ariidae e Gerreidae, há, entre os peixes abundantes, pelo menos uma espécie de Carangidae (C. chrysurus) e um Sciaenidae (M. furnieri), que é mais abundante em zonas subtropicais e temperadas. Segundo Stoner (1986), os Sciaenidae substituem os Gerreidae como espécie demersal dominante nas zonas temperadas. A grande ocorrência de Gerreidae tem sido comprovada em outras baías e lagoas costeiras do Estado do Rio de Janeiro, onde os juvenis estão associados à margem continental, que é o ambiente natural para o desenvolvimento dos primeiros estágios de vida (Andreata, 1987; Silva, 1994; Araújo, 1997).

Embora seja difícil comparar a riqueza de espécies da ictiofauna devido à heterogeneidade de hábitats, diferenças físico-químicas no ambiente, bem como diferenças no esforço de pesca, está evidenciado que a Baía de Sepetiba apresenta um elevado número de espécies comparativamente com outras regiões do Sudeste do Brasil. Andreata et al. (1990) encontrou 49 espécies para a Laguna da Tijuca, RJ; Brum et al. (1994), 34 espécies para o sistema lagunar de Maricá.

A relativa estabilidade dos fatores ambientais da Baía de Sepetiba, principalmente no que concerne à salinidade, pode ter propiciado o desenvolvimento desta fauna bastante rica em espécies. A transparência da água, maior na zona próxima do encontro com o mar, e menor na região mais interior e protegida da Baía, pode estar associada à maior abundância de peixes na zona mais interna, onde a maior concentração de nutrientes provenientes da drenagem continental referida por Costa (1992) estaria contribuindo para a maior produtividade nesta zona.

O maior número total de espécies e de espécie/amostra registrado nas estações da zona externa da Baía indica que as espécies se substituem ao longo do ano, especialmente os visitantes cíclicos marinhos, que provavelmente encontram nesta zona melhores condições ambientais e menor número de competidores quando comparado com a zona interna e protegida da Baía. Também o nível de estresse parece ser maior na zona interna, devido ao fundo de natureza mais lodoso, pela proteção do cordão de ilhas formado a partir da 
Ilha de Itacuruçá, tornando a zona mais calma, e à maior proximidade de centros urbanos, que carreiam para aquela estação maior contribuição de matéria orgânica.

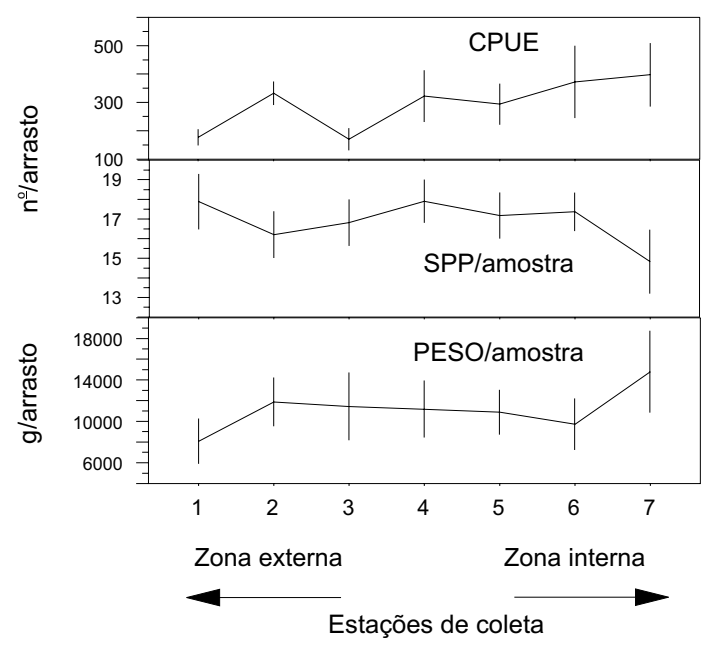

Fig. 6 - Capturas por unidade de esforço (CPUE), número espécies/arrasto e peso/arrasto de peixes coletados na Baía de Sepetiba, RJ, entre julho de 1993 e junho de 1994. Barras representando +/- 1 erro padrão.

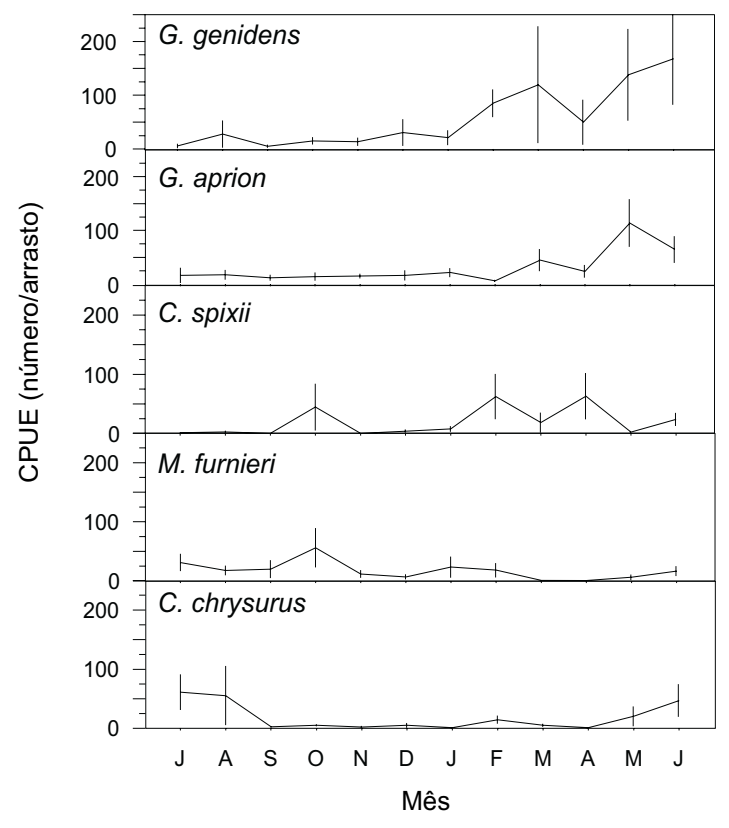

Fig. 7 - Variação temporal nas capturas por unidade de esforço (CPUE) das cinco espécies de peixes mais abundantes, na Baía de Sepetiba, RJ, entre julho de 1993 e junho de 1994.

Na zona interna da Baía, onde as condições ambientais parecem ser mais estressantes, o nú- mero total de espécies registradas, e o número de espécies/amostra foram menores. Por outro lado, as maiores CPUEs e peso/amostra nessa zona indicam o eficiente uso dos recursos alimentares deixados disponíveis pelos peixes, com a ictiofauna sendo associada às condições de baías e lagoas costeiras, como foi o caso dos Ariidae G. genidens e C. spixii e do Carangidae C. chrysurus.

Indicações de diferenças na estrutura da comunidade de peixes entre a zona interna e externa da Baía, especialmente nas estações 3 e 4 (Ilha Bonita e Costa do Guandu), que se apresentaram mais similares às outras estações de coleta, caracterizando uma situação de fauna intermediária entre as duas zonas da Baía, foi confirmada pelo coeficiente de similaridade de Czekanowski. Tal coeficiente apresentou maiores similaridades dentre as estações da zona externa de Ilha do Socó e Laje das Enxadas (estações 1 e 2) e dentre as estações mais internas de Canal da Restinga, Meio da Baía e Fundo da Baía (estações 5,6 e 7).

Vários autores têm estabelecido (Gunther, 1958; McFarland, 1963; Saloman \& Naughton, 1979; Cunha, 1981) que as maiores abundâncias de peixes em zonas costeiras, de regiões subtropicais e temperadas quentes, ocorrem no verão, e as menores, no inverno. Na Baía de Sepetiba, localizada numa área de transição entre a região subtropical e temperada quente, os padrões sazonais de abundância da ictiofauna foram pouco evidentes.

Muitos dos peixes mais abundantes na Baía de Sepetiba apresentaram padrão de distribuição espacial mais definido, como foi o caso de $G$. genidens, G. aprion e M. furnieri. Saul (1994), estudando a comunidade ictiofaunística na região de Cananéia, SP, constatou a existência de padrões espaciais na comunidade de peixes, associados principalmente à diferenciação entre os hábitats e padrões sazonais evidentes.

Na Baía de Sepetiba, embora as variações sazonais não tenham sido bem caracterizadas, as maiores CPUEs entre fins do verão e início do inverno sugerem o recrutamento dos peixes provenientes das desovas do verão. O padrão espacial foi melhor definido do que o sazonal, com as CPUEs e picos de espécies mais comuns ocorrendo em locais diferenciados, denotando a separação espacial como estratégia de coexistência. O elevado número de espécies encontrado sugere que 


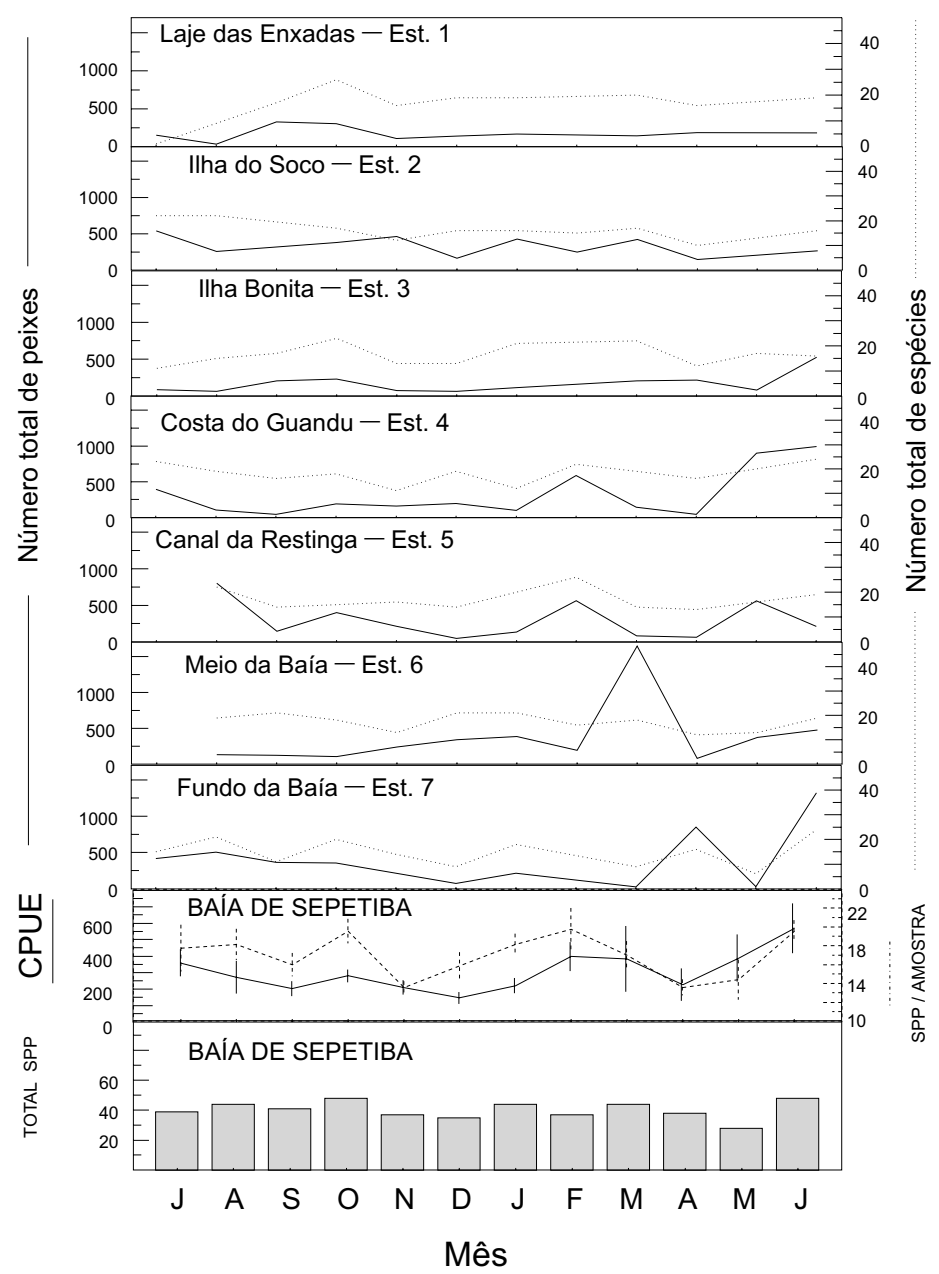

Fig. 8 - Variação temporal e espacial da abundância relativa de peixes (CPUE) e número de espécies na Baía de Sepetiba, RJ.

muitos peixes utilizam a Baía como área de criação durante parte ou todo o ciclo de vida.

Agradecimentos - Aos estagiários do Laboratório de Ecologia de Peixes do Posto de Aqüicultura da Univ. Fed. Rural do Rio de Janeiro, Iracema David Gomes, Ingrid Castro Bertoldo, André Luiz Machado Pessanha, Márcio de Araújo Silva, Pablo Mendonça e Marcelo Sales Moffati, pelo apoio nos trabalhos de campo e de laboratório. Também agradecemos o suporte recebido do Conselho Nacional de Desenvolvimento Científico e Tecnológico - CNPq, através de auxílio e bolsas.

\section{REFERÊNCIAS BIBLIOGRÁFICAS}

AMEZCUA-LINARES, F. \& YÁÑEZ-ARANCIBIA, A., 1980, Ecologia de los sistemas fluvio-lagunares associados a la laguna de Términos. An. Centro Cienc. del Mar y Limnol., Univ. Nal. Auton. México, 7(1): 69-118.
ANDREATA, J. V., 1987, Revisão das espécies de Gerreidae (PERCIFORMES, PERCOIDEI) que ocorrem no litoral brasileiro e relações entre os gêneros. Tese de Doutorado do Inst. de Biociências da Univ. de São Paulo, São Paulo, 233p.

ANDREATA, J. V., 1988, Revisão taxonômica do Gênero Diapterus Ranzani 1840 (Pisces, Perciformes, Gerreidae). Acta Biol. Leopoldensia, 10(1): 59-103.

ANDREATA, J. V., SAAD, A. M. \& BARBIERI, L. R., 1989, Associação e distribuição das espécies de peixes na laguna de Marapendi, Rio de Janeiro, no período de março de 1985 a fevereiro de 1987. Mem. Inst. Oswaldo Cruz, 84(suppl. IV): 45-51.

ANDREATA, J. V., SAAD, A. M., BIZERRIL, C. R. S. \& BOCKMANN, F. A., 1990, Alguns aspectos da ecologia das espécies de peixes da Laguna da Tijuca, período de março de 1987 a fevereiro de 1989. Acta Biol. Leopoldensia, 12(2): 247-268. 
ARAÚJO, F. G., CRUZ-FILHO, A. G., AZEVÊDO, M. C., SANTOS, A. C. A. \& FERNANDES, L. A. M., 1997, Estrutura da comunidade de peixes jovens da margem continental da Baía de Sepetiba, RJ. Acta Biol. Leopoldensia, 19(1): 61-83.

BLABER, S. J. M., BREWER, D. T., SALINI, J. P., KERR, J. D. \& CONACHER, C., 1992, Species composition and biomass of fishes in tropical seagrasses at the Groote Eylandt, Northern Australia. Estuar. Coast. Shelf Science, 35(6): 605-620.

BRAY, J. R. \& CURTIS, J. T., 1957, An ordination of the upland forest communities of southern Wisconsin. Ecol. Monogr., 27: 325-345.

BRUM, M. J. I., MURATORI, C. F. M., LOPES, P. R. D. \& VIANNA, P. R. F., 1994, Ictiofauna do sistema lagunar de Maricá (RJ). Acta Biol. Leopoldensia, 16(2): 45-55.

COELHO, V. M. B. \& CARVALHO, R. R., 1973, Levantamento sanitário da Baía de Sepetiba e suas possibilidades como corpo receptador de cargas poluidoras da Região. Publicações Avulsas FEEMA, Rio de Janeiro, 87p.

COSTA, R. N. L. T. R., 1992, Pensar o mar para poder pescar: o espaço da pesca de litoral na Baía de Sepetiba, RJ. Tese de Mestrado do Curso de Pós-Graduação em Geografia da Univ. Fed. Rio de Janeiro, Rio de Janeiro, $181 \mathrm{p}$.

CRUZ-FILHO, A. G., 1995, Variações espaciais e temporais na comunidade de peixes da Baía de Sepetiba, RJ. Tese de Mestrado do Curso de Pós-Graduação em Ciências Ambientais e Florestais da Univ. Fed. Rural do Rio de Janeiro, Rio de Janeiro, 99p.

CUNHA, L. P. R., 1981, Variação sazonal da distribuição, abundância e diversidade dos peixes na zona de arrebentação da Praia do Cassino, RS, Brasil. Tese de Mestrado em Zoologia da Univ. Fed. do Rio de Janeiro, Rio de Janeiro, 47p.

CUNNINGHAM, P. T. M., 1983, Estudo comparativo da ictiofauna da costa Oeste e enseada das Palmas, Ilha Anchieta, Enseada do Flamengo e Enseada da Fortaleza

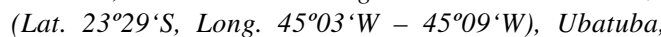
Estado de São Paulo, Brasil. Tese de Doutorado do Inst. Oceanográfico da Univ. de São Paulo, São Paulo, 133p.

FIGUEIREDO, J. L., 1977, Manual dos peixes marinhos do Sudeste do Brasil. I. Introdução. Cações e raias e quimeras. Museu de Zoologia, Univ. de São Paulo, São Paulo, $104 \mathrm{p}$.

FIGUEIREDO, J. L. \& MENEZES, N. A, 1978, Manual dos peixes marinhos do Sudeste do Brasil. II. Teleostei (1). Museu de Zoologia, Univ. de São Paulo, São Paulo, 110p.

FIGUEIREDO, J. L. \& MENEZES, N. A., 1980, Manual de peixes marinhos do Sudeste do Brasil. III. Teleostei (2). Museu de Zoologia da Univ. de São Paulo, São Paulo, 90p.

FISCHER, W. (ed.)., 1978, FAO species identification sheets for fishery purposes. Western Central Atlantic (Fishing Area 31). Vol I-VII. Roma, FAO, sem paginação.

GUNTHER, G., 1958, Population studies of the shallow water fishes of an outer beach in south Texas. Public. Inst. of Mar. Sciences., Univ. of Texas., 5: 186-193.
HOOK, J. H., 1991, Seasonal variation in the relative abundance and species diversity of fishes in South Bay. Contr. Mar. Sci., 32: 127-141.

LIVINGSTON, R. J., KOBYLINSKI, G. J., LEWIS, F. G. \& SHERIDON, P. F., 1976, Long-term fluctuations of epibenthic fish and invertebrate populations in Apalachiola Bay, Florida. Fishery Bull., 74: 311-321.

McFARLAND, W. N., 1963, Seasonal change in the number and the biomass of fishes from the surf at Mustang Island, Texas. Public. Inst. of Mar. Sciences, Univ. of Texas, 9: 91-112.

MENEZES, N. A. \& FIGUEIREDO, J. L., 1980, Manual de peixes marinhos do Sudeste do Brasil. IV. Teleostei (3). Museu de Zoologia, Univ. de São Paulo. São Paulo, 96p.

MENEZES, N. A. \& FIGUEIREDO, J. L., 1985, Manual de peixes marinhos do Sudeste do Brasil. V. Teleostei (4). Museu de Zoologia, Univ. de São Paulo. São Paulo, 105p.

MONTEIRO-NETO, C., BLACHER, C., LAURENT, A. S., SNIZEK, F. N., CANOZZI, M. B. \& TABAJARA, L. L. C., 1990, Estrutura da comunidade de peixes de águas rasas na região de Laguna, Santa Catarina, Brasil. Atlântica, 12(2): 53-69.

NORMAN, J. R., 1934, A systematic monography of the flatfishes (Heterosomata). I. Psettodidae, Bothidae, Pleuronectidae. Johnson Repr. London, pp. 59-261.

OLIVEIRA, S. S., 1988, Aspectos sazonais de ocorrência, tamanho e dieta de duas espécies de Engraulididae (Teleostei, Clupeiformes) na Baía de Sepetiba, RJ. Tese de Mestrado em Zoologia da Univ. Fed. do Rio de Janeiro, Rio de Janeiro, 97p.

OSHIRO, L. M. Y. \& ARAÚJO, F. G., 1987, Estudo dos peixes e crustáceos decápodes da Baía de Sepetiba, RJ. Simpósio Ecossistemas Costeiros do Sudeste-Sul do Brasil. Acad. Ciênc. São Paulo, São Paulo, 3(54): 283-297.

PFEIFFER, W. C., LACERDA, L. D., FISZMAN, M. \& LIMA, N. R., W., 1985, Metais pesados no pescado da Baía de Sepetiba. Ciênc. e Cultura, 37(2): 297-302.

SALOMAN, C. H. \& NAUGHTON, S. P., 1979, Fishes of the littoral zone, Pinellas County, Florida. Florida Sciences, 42(2): 85-93.

SAUL, A. C., 1994, Comunidade ictiofaunística da Ilha do Bom Abrigo, Cananéia, SP, Brasil. Tese Mestrado do Inst. Oceanográfico da Univ. São Paulo, São Paulo, 118p.

SEDBERRY, G. R. \& CARTER, J., 1993, The fish community of a shallow tropical lagoon in Belize, Central America. Estuaries, 16(2): 198-215.

SILVA, M. H. C., 1994. Ocorrência de Gerreidae (Osteichthyes, perciformes) no canal de Camboatá, sistema lagunar de Itaipu, Piratininga, RJ. Abundância relativa e cronologia alimentar. Tese de Mestrado em Zoologia da Univ. Fed. do Rio de Janeiro, Rio de Janeiro, 168p.

SOKAL, R. R. \& ROHLF, F. J., 1981, Biometry; the principles and practice of statistics in biological research. W. H. Freeman and Co., San Francisco, 776p. 
SOUZA, D. C. \& ARAÚJO, F. G., 1990, Distribuição e abundância do peixe-rei Xenomelaniris brasiliensis (Quoy \& Gaimard, 1824) na Baía de Sepetiba, RJ. IBAMA, Serviço de Defesa Ambiental SDA, 7: 1-12.

STONER, A. W., 1986, Community structure of the demersal fish species of Laguna Joyuda, Puerto Rico. Estuaries, 9(2): 142-152.

VOLCKER, C. M. \& ANDREATA, J. V., 1982, Levantamento taxonômico preliminar da ictiofauna da laguna da Tijuca, Rio de Janeiro. Rev. Nordest. Biol., 5(2): 197-257.

VILLARROEL, P. R., 1994, Estructura de las comunidades de peces de la laguna de Raya, Isla de Margarita, Venezuela. Ciencias Marinas, 20(1):1-16.
WRIGHT, J. M., 1991, Annual differences in the fish assemblage of the non-estuarine Sulaibikhant Bay, Kuwait. In: M. Elliot \& J. P. Ducrotoy (eds.) Estuaries and coasts: spatial and temporal intercomparisons, milleux estuaries et littoraux. New York, pp. 285-288.

YÁÑEZ-ARANCIBIA, A., AMEZCUA-LINARES, F. \& DAYJR, J. W., 1980, Fish community structure and function in Terminos Lagoon, a tropical estuary in the southern Gulf of Mexico. In: V. S. Kennedy (ed.). Estuarine Perspectives. Academic Press, New York, pp. 465-482. 